\title{
Nouvelles Pascaliennes
}

Dominique Descotes, CERHAC et Université Blaise Pascal

\section{OpenEdition \\ Journals}

Édition électronique

URL : http://journals.openedition.org/ccibp/439

DOI : $10.4000 /$ ccibp.439

ISSN : 2493-7460

\section{Éditeur}

Centre international Blaise Pascal

\section{Édition imprimée}

Date de publication : 5 avril 1985

Pagination : 20-22

ISSN : 0249-6674

Référence électronique

Dominique Descotes, CERHAC et Université Blaise Pascal, « Nouvelles Pascaliennes », Courrier du

Centre international Blaise Pascal [En ligne], 7| 1985, mis en ligne le 27 novembre 2015, consulté le 23 avril 2019. URL : http://journals.openedition.org/ccibp/439 ; DOI : 10.4000/ccibp.439

Ce document a été généré automatiquement le 23 avril 2019

Centre international Blaise Pascal 


\title{
Nouvelles Pascaliennes
}

\author{
Dominique Descotes, CERHAC et Université Blaise Pascal
}

\section{L'animation scientifique en Auvergne}

1 On se souvient des expériences du vide et notamment de la montée au Puy-de-Dôme que M. Roland JOUANISSON, Maître de conférences à l'Université II de Clermont-Ferrand, avait réalisées pour les journées du CIBP en octobre 1981. Aujourd'hui notre ami et correspondant nous annonce la naissance d'une Association pour le Développement de l'Animation Scientifique et Technique en Auvergne, soit ADASTA, qui a pour but de diffuser et promouvoir la culture scientifique et technique dans la région Auvergne.

2 Cette association vise particulièrement les jeunes qu'elle touche par le moyen des associations culturelles et par l'action dans le milieu industriel. Monsieur Roger Vessière, Directeur de l'École nationale de Chimie de Clermont-Ferrand, en assure la présidence, et M. Roland Jouanisson la direction scientifique. Elle comprend des membres fondateurs et des adhérents, actifs, correspondants, bienfaiteurs.

3 L'association des Amis et Correspondants du CIBP souhaite une heureuse carrière à cette jeune amie, dont l'objectif corrobore sa propre vocation, scientifique avec un ancrage régional résolu: Blaise Pascal "Auvergnat », et géomètre, soucieux comme il l'était de joindre l'ouverture sociable, qu'il appelait «l'honnêteté», à l'ardeur de connaître, n'aurait pas manqué de s'inscrire à l'ADASTA...

4 Cotisations pour les particuliers : 50 F. Pour les collectivités, entreprises, etc. $500 \mathrm{~F}$.

5 Adresser adhésions et correspondance à M. R. Jouaisson, Laboratoire d'Électromagnétisme, B.P. 45, 63170 Aubière. 


\section{On recherche la famille...}

6 Et l'on a quelques moyens de plus pour cette recherche avec une table des mariages de l'Ancien Régime en Auvergne, dressé par l'érudit L. Le Peletier d'Aulnay, à travers le fonds Keating. Photocopie déposée au CIBP. Une feuille recto/verso.

7 Autres photocopies intégrées:

8 •C. J. A. Chapot-Laroche: «Mémoires ou Notes généalogiques sur la famille ChapotLaroche », 1825, 2 p.

- Raymond Josse : « Pierre Pascal, prêtre du diocèse de Clermont-en-Auvergne », 2 p.

Les Amis du CIBP sont en liaison avec l'ARGHA (Association de Recherches généalogiques et historiques d'Auvergne, Maison des Consuls, Place Poly, 63100 Clermont-Ferrand). Deux lignes de continuité en association, cela peut faire une force... de recherche, si tous ceux qui se souviennent d'avoir un point de contiguïté avec la famille de Blaise Pascal veulent bien se donner la peine de remonter leur ascendance jusqu'à ce point.

11 La Présidente de l'ARGHA nous a promis une recherche sur Mathie PASCAL, cousine germaine de Blaise, veuve en premières noces de Guillaume de Grandsaigne, puis thiernoise comme épouse, en 1681, de Gilbert Bérard.

\section{La vie de l'association de soutien}

L'assemblée générale statutaire de l'association Amis et Correspondants du CIBP s'est tenue à la Bibliothèque municipale et interuniversitaire (BMIU) de Clermont-Ferrand, salle Massillon, le vendredi 13 décembre 1985, à $17 \mathrm{~h}$, sous la présidence de la présidente, $\mathrm{M}^{\mathrm{me}}$ Goyet.

13 Le compte-rendu de l'assemblée du 12 décembre 1984, lu par la secrétaire de l'association, $\mathrm{M}^{\text {elle }}$ Marie-Thérèse Sart a été approuvé à l'unanimité.

14 Le rapport financier présenté par le trésorier, M. Dominique Descotes, a été approuvé à l'unanimité, moins une abstention.

\section{Rapport moral}

$\mathrm{M}^{\mathrm{me}}$ Goyet, commence par la comparaison avec l'état de l'association à la même date l'année précédente. Pour elle-même la différence est grande en raison de son admission à la retraite et de son installation à Paris. Heureusement M. Descotes, maître de conférences à l'Université Clermont II et docteur-ès-lettres, assure la continuation de l'enseignement et de la recherche concernant Pascal, et la Bibliothèque Municipale et Interuniversitaire qui nous contient est la garantie de notre durée : la mémoire propre d'une civilisation dépend de tels organismes. Il est vrai que la recherche des traces concrètes laissées par Pascal et les siens, que nous avons appelée « itinéraire pascalien en Auvergne ", est difficile à mener loin de l'Auvergne, mais de nombreux points d'insertion ont déjà été marqués, où les Amis du CIBP peuvent prendre leur ancrage afin de développer une connaissance qui sera multiforme et qui peut apporter de l'inattendu. 
16 Le Courrier $n^{\circ} 6$, millésimé 1984 , a été réalisé selon le projet annoncé. Il apporte une contribution inédite et fort importante à l'iconographie de Pascal, et sa présentation élégante a été appréciée.

Les deux événements majeurs de l'exercice précédent ont été d'une part les deux sessions du colloque scientifique et pédagogique "L'accès aux Pensées de Pascal ", qui se sont tenues au CRDP les 7-9 novembre 1984 et 13-15 mai 1985, et l'acquisition par la Ville de Clermont-Ferrand d'une seconde machine arithmétique conservée jusqu'ici dans une famille d'Auvergne. Le colloque marqué par la participation chaleureuse des professeurs de l'Académie, a bien fait voir le rapport existant entre la culture scolaire, ouverture première, et la vie spirituelle d'une société. Le CIBP s'est engagé dans la publication des Actes. L'intronisation de la pascaline au Musée du Ranquet, présentée à la presse le 29 octobre dernier et fort bien commentée par les publications municipales, signifie l'engagement cordial de la Ville et de l'État dans la promotion des souvenirs de Pascal. Cette réussite vient à propos pour «échauffer », comme dit Pascal, le zèle des Amis du CIBP.

18 Les projets sortent naturellement de l'esprit de famille qui nous anime. Nous avons à rechercher notamment :

- un portrait (signalé) de Florin Périer ;

- le sens des armes de Pascal trouvées sur une pierre ;

- des compléments sur la maison de l'Oratoire (voir Courrier $n^{\circ} 5$ ) ; l'emplacement exact de la maison de ville de Florin Périer, Bien-Assis, maison des champs, étant maintenant connu ( Courrier $\mathrm{n}^{\circ} 3$ et 4 ).

19 Pour sa part la Présidente souhaite mener aussi loin que possible le dépouillement du fonds de Keating, fonds privé afférant à l'hôtel, 12, rue Pascal, que la Ville a acheté en raison de ses peintures du $17^{\mathrm{e}}$ siècle. Clermont, du $16^{\mathrm{e}}$ au $19^{\mathrm{e}}$ siècle s'y découvre comme le monde où se sont insérés les Pascal.

Le contenu du Courrier $n^{\circ} 7$ sera déterminé dans quelques jours par la réunion du Conseil de gestion.

21 La recherche du film de Rossellini et celle du portrait de Pascal ayant appartenu à Charles Silvestre n'ont hélas pas progressé malgré de nouvelles démarches. Mais nos amis dans leurs initiatives savent intéresser d'autres organismes culturels; ainsi M. Mourlevat engage le CRDP et l'Académie des Sciences, Belles-Lettres et Arts de Clermont dans une publication sur les machines arithmétiques qu'il entreprend, et M. Yves Morvan se lance dans de nouvelles recherches iconographiques. Ce développement sur le mode fédératif est on ne peut plus intéressant et il convient d'encourager chacun à saisir sa propre occasion et à la poursuivre jusqu'au bout.

Le rapport moral est approuvé à l'unanimité moins une abstention.

\section{Élection}

$M^{\text {elle }}$ Sart rappelle l'article 10 des statuts selon lequel le tiers des membres du Conseil doit être renouvelé. Le sort désigne pour le départ M. Gautier de Breuvant, $M^{\text {elle }}$ Seguin et $M$. Sellier. Le nombre des conseillers n'étant pas déterminé, il a paru utile de susciter quatre candidatures : M. René Combe, M. Philippe Gautier de Breuvant, M. Guy Mourlevat et M elle $^{\text {. }}$ Dominique Seguin. Chaque candidat est élu à l'unanimité. Le Bureau répartit immédiatement entre eux les fonctions suivantes : 
$$
1986
$$
1986.

M. Mourlevat : vice-président ; $M^{\text {elle }}$ Seguin : trésorier-adjoint ;

M. Gautier de Breuvant : correspondant généalogiste.

\section{Cotisation}

Il est jugé bon de la laisser pour 1986 à $60 \mathrm{~F}$ pour les membres actifs, $200 \mathrm{~F}$ ou davantage pour les membres bienfaiteurs.

\section{Questions diverses, suggestions, vœux}

$\mathrm{M}^{\text {me }}$ Sauvadet représentant l'Association des Recherches Généalogiques en Auvergne souhaite qu'on lui prépare une note sur les généalogies Pascal, qu'elle diffusera volontiers.

$\mathrm{M}^{\text {elle }}$ Lasbats, responsable de la Chancellerie des Universités, représentant M. le Recteur, fait une suggestion pour obtenir l'aide des media.

Le CIBP collaborera à la publicité pour l'ouvrage que l'Académie projette d'éditer sur les machines arithmétiques de Pascal.

On se plaint des frais postaux élevés.

$\mathrm{M}^{\text {elle }}$ Leclercq, Directeur des Archives Départementales nous engage à participer au Congrès des Sociétés savantes du Centre qui se tiendra à Clermont-Ferrand les 9 et 10 mai

On demande instamment à la Municipalité que le petit monument à l'emplacement de la maison natale de Pascal soit entretenu décemment et surtout protégé des chiens.

L'association souhaite ardemment que le projet du musée consacré à Pascal soit mené à bien dans les meilleurs délais.

P.S. Le compte-rendu de l'A.G du 18 décembre 1986 sera publié dans le Courrier $n^{\circ}$ 8, sous le millésime 1986, qu'on espère réaliser avec un moindre décalage dans le temps.

BIBLIOGRAPHIE

\section{Liste des dons reçus par le CIBP entre le $1^{\text {er }}$ juillet 1985 et le 30 juin 1986}

\section{Documents imprimés}

AUGARTEN Stan, « Bit by bit: an illustrated history of computers, » New York, 1984.

Les Auvergnats : $\mathrm{n}^{\circ} 88$ de Le Crapouillot, 1986.

BEAUREPAIRE Charles de, « Blaise Pascal et sa famille à Rouen de 1640 à 1647 » Rouen, 1902. 
Dossier de presse concernant l'achat par la Ville de Clermont de la machine arithmétique DurantPascal. 1985.

Études sur Pascal, 1623-1923, Paris, 1923.

FERREYROLLES Gérard, « Le Prince selon Pascal » Paris, 1985.

L'Illustration : $\mathrm{n}^{\circ}$ du samedi 16 juin 1923.

Le Jansénisme dans l'Yonne : catalogue d'exposition, Auxerre, 1986.

Jansénius et son temps : catalogue d'exposition, Louvain, 1985.

MAIRE Albert, Essai bibliographique des « Pensées », Paris, 1923.

MASSIS Henri, Troisième centenaire de la mort de Pascal célébré à Rouen le 10 novembre 1962 : discours. Paris, 1962.

MESNARD Jean, « Le Drame de la foi chez Pascal », Paris, 1958.

PARCÉ Léon, « La Réimpression des premières Provinciales », 1963 (épreuves dactyl.)

Pascal : n 63 des « Grands écrivains », 1986.

PLAINEMAISON Jacques, « Qu'est-ce que le jansénisme ? », Paris, 1985.

Programme de la saison théâtrale de 1986 du théâtre Gérard Philippe de Saint-Denis ; avec le texte de l'« Entretien de M. Descartes avec M. Pascal le jeune » par J.-C. Brisville. Paris, 1986.

Les Provinciales : arts, culture et société : nº 3. Clermont, 1986.

QUILLIOT Roger, « Un exemple d'influence pascalienne au $20^{\mathrm{e}}$ siècle », L'œuvre d'Albert Camus, Clermont, 1971.

Sardines à l'instar : pastiches littéraires par un escholier de Louvain, Paris, 1953.

SUEMATSU Hisashi, «Lire les Pensées : la relation binaire comme invariant », Tokyo, 1985.

Troisième centenaire de Pascal : $\mathrm{n}^{\circ}$ de la Revue hebdomadaire, 1923.

\section{Documents iconographiques}

Élévation de la Montagne du Puy-de-Dôme : planche gravée extraite de l’Encyclopédie de Diderot.

\section{Publications en dépôts au CIBP}

Le catalogue de l'exposition Blaise Pascal « Auvergnat ». Clermont-Ferrand, 1981, 110 p., 18 pl., fig. Franco, 53 F.

Quatre cartes postales reproduisant les filigranes du papier utilisé par Pascal. Franco, 12,50 F.

« Deux grandes figures d'Auvergne. Gilberte et Jacqueline Pascal ». Chroniques de Port-Royal, $\mathrm{n}$

- 31, Clermont-Ferrand, 1982, 158 p., 9 pl. Franco, 121 F.

«Ordre par dialogues ». Récital des Pensées donné le 14 mai 1985. Une cassette, prise au bureau $20 \mathrm{~F}$. Envoi par la poste $25 \mathrm{~F}$.

« La Violence et la Vérité ». Les Provinciales de Blaise Pascal. Récital donné le 16 mai 1986. Une cassette. Prise au bureau $20 \mathrm{~F}$. Envoi par la poste $25 \mathrm{~F}$. 